\title{
Epithelial cell transforming sequence 2 expression is associated with the progression of laryngeal squamous cell carcinoma
}

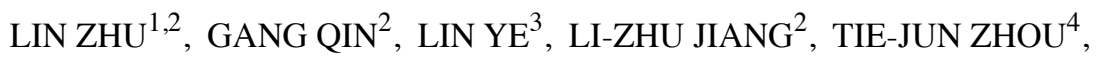 \\ BO LUO ${ }^{5}$, TAO-YU WEN ${ }^{1}$, YA-LAN LIU ${ }^{1}$ and HONG-YAN CHEN ${ }^{1}$

\begin{abstract}
${ }^{1}$ Department of Otolaryngology, Head and Neck Surgery, The First Affiliated Hospital of Chongqing Medical University, Chongqing 400016; ${ }^{2}$ Department of Otolaryngology, Head and Neck Surgery, The Affiliated Hospital of Southwest Medical University, Luzhou, Sichuan 646000; ${ }^{3}$ Department of Cardio-Thoracic Surgery, The First Hospital of Southwest Medical University; ${ }^{5}$ Department of Biochemistry, Institute for Cancer Research, School of Preclinical Medical Sciences, Southwest Medical University, Luzhou, Sichuan 646000, P.R. China
\end{abstract} \\ Affiliated Hospital of Chongqing Medical University, Chongqing 400016; ${ }^{4}$ Department of Pathology, The Affiliated
}

Received September 3, 2018; Accepted February 25, 2019

DOI: $10.3892 / \mathrm{ol} .2019 .10226$

\begin{abstract}
Epithelial cell transforming sequence 2 (ECT2) is a guanine nucleotide exchange factor and its expression is associated with the development of malignant tumor types. However, to the best of our knowledge, there is no information on the role of ECT2 in the development and progression of laryngeal squamous cell carcinoma (LSCC). The present study aimed at investigating the expression pattern and potential role of ECT2 in the development and progression of LSCC. The expression of ECT2 in 81 pairs of LSCC and adjacent non-tumor tissues was characterized by immunohistochemistry and reverse transcription-quantitative polymerase chain reaction. ECT2 expression was upregulated in LSCC tissues and associated significantly with poor differentiation, higher stages, lymph node metastasis and poor survival in the sample population. The relative expression levels of ECT2 mRNA transcripts were correlated with the intensity of anti-ECT2 staining in 25 ECT2+LSCC specimens selected randomly. These results indicated that ECT2 expression was crucial for the progression of LSCC and may serve as a biomarker for the diagnosis and prognosis of LSCC.
\end{abstract}

\section{Introduction}

Laryngeal cancer is one of the most common malignancies in the respiratory system globally (1), and the number of new

Correspondence to: Dr Hong-Yan Chen, Department of Otolaryngology, Head and Neck Surgery, The First Affiliated Hospital of Chongqing Medical University, 1 Youyi Road, Chongqing 400016, P.R. China

E-mail: chy201788@163.com

Key words: laryngeal squamous cell carcinoma, epithelial cell transforming sequence 2, immunohistochemistry, reverse transcription-quantitative polymerase chain reaction, poor survival cases of laryngeal cancer has reached 3.0 per 100,000 people annually between 2011 and 2015 due to excessive tobacco and alcohol consumption (2-4). Furthermore, exposure to asbestos, dust and polycyclic aromatic hydrocarbons is associated with an increased risk of laryngeal squamous cell carcinoma (LSCC) development $(3,5,6)$. Despite the fact that therapeutic strategies, including surgical techniques, chemotherapeutic drugs and radiotherapy, have advanced, the 5-year overall survival rate of patients with LSCC remains as low as 61\% (7). The pathogenesis of LSCC remains unclear and understanding the factors contributing to the development and progression is crucial for the discovery of novel therapeutic targets, in addition to the management of patients with LSCC.

Epithelial cell transforming sequence 2 (ECT2) is a guanine nucleotide exchange factor and can regulate the process of cell cycling and cytokinesis (8). ECT2 contains two BRCA1 C Terminus (BRCT) domains within the N-terminus, including a DBL-homology domain and a pleckstrin homology domain (9). Previous studies reported that ECT2 is localized in the actin cortex and central spindles, as ECT2 is crucial for the cleavage furrow formation during cytokinesis $(10,11)$. The upregulated ECT2 expression and its misplacement have been indicated to be associated with the tumorigenesis and the development of numerous malignant tumors, including lung cancer, ovarian cancer and primary brain cancer (12-14), in addition to poor survival in patients with colorectal cancer or lung adenocarcinoma $(15,16)$. However, to the best of our knowledge, there is no information on the levels of ECT2 expression and its role in the development and progression of LSCC.

In the present study, ECT2 expression was analyzed in 81 LSCC specimens and adjacent non-tumor tissues, and the association between the levels of ECT2 expression and clinical characteristics of patients with LSCC was examined.

\section{Patients and methods}

LSCC tissue specimens. A total of 81 patients (79 males and 2 females, median age of 61.3 years old, ranging 
from 41 to 76 years old) with LSCC were recruited at the in-patient service of the Department of Otolaryngology, Head and Neck Surgery of The Affiliated Hospital of SouthwestMedical University (Luzhou, China) between September 2012 and March 2015. Individual patients with LSCC were diagnosed and staged, according to the criteria of the Union for International Cancer Control and the Tumor-Node-Metastasis (TNM) classification system (2012) (17). Patients underwent partial (62 patients) or total laryngectomy (19 patients), and their respective LSCC samples were collected. The inclusion criteria included that individual patients had complete clinical data and did not receive antitumor radiotherapy and chemotherapy. Otherwise, individual patients with incomplete medical record or received any preoperative antitumor treatment were excluded. These 81 pairs of human LSCC and their adjacent non-tumor tissue specimens were used for immunohistochemistry. Finally, 25 pairs of human LSCC and their adjacent non-tumor tissues were randomly selected for reverse transcription-quantitative polymerase chain reaction (RT-qPCR), based on positive ECT2 expression by immunohistochemistry. Written informed consent was obtained from individual subjects participating in the present study. The experimental protocol was approved by the Ethnic Committee of The Affiliated Hospital of Southwest Medical University. One part of the tissue samples was fixed in $10 \%$ neutral buffered formalin for $24 \mathrm{~h}$ at room temperature and paraffin-embedded. The remaining tissues were immediately frozen in liquid nitrogen, and stored at $-80^{\circ} \mathrm{C}$ until RNA and protein extraction.

The 81 patients with LSCC were followed-up by telephone communication or out-patient visiting until April 31, 2018. Their overall survival time was defined as the time between surgery and mortality from LSCC.

$R T$ - $q P C R$. Total RNA was extracted from individual tissue samples with TRIzol ${ }^{\circledR}$ (Invitrogen; Thermo Fisher Scientific, Inc., Waltham, MA, USA), according to the manufacturer's protocols, and the RNA ( $1 \mu \mathrm{g}$ per sample) was reversely transcribed into cDNA using a PrimeScript ${ }^{\mathrm{TM}}$ RT-PCR kit (Takara Bio, Inc., Otsu, Japan), according to the manufacturer's protocols. The relative expression levels of ECT2 to the control GAPDH mRNA transcripts were determined by RT-qPCR using a SYBR ${ }^{\mathrm{TM}}$ Green PCR Master mix (Applied Biosystems; Thermo Fisher Scientific, Inc.) and specific primers. The sequences of the primers were as follows: ECT2 (119 bp) forward, 5'-GCCATAATGCTTTTGCCTTGCTTG-3' and reverse 5'-TCGACACAGCATCTTTAGCCAGTTT-3'; GAPDH (177 bp) forward, 5'-GGGCCAAAAGGGTCATCA TCTC-3'and reverse 5'-GCCCTTCCACGATGCCAAA-3'. The PCR reactions were performed in triplicate and the thermocycling conditions were as follows: $95^{\circ} \mathrm{C}$ for $5 \mathrm{~min}$; and 40 cycles of $95^{\circ} \mathrm{C}$ for $30 \mathrm{sec}, 57^{\circ} \mathrm{C}$ for $30 \mathrm{sec}$ and $72^{\circ} \mathrm{C}$ for $30 \mathrm{sec}$. The data were analyzed by the $2^{-\Delta \Delta \mathrm{Cq}}$ method (18).

Immunohistochemistry. The tissues were fixed in $10 \%$ neutral buffered formalin for $24 \mathrm{~h}$ at room temperature and then dehydrated in an ascending ethanol series and cleared with 3 changes of xylene, followed by paraffin-embedding. The paraffin-embedded tissue sections (thickness, $4 \mu \mathrm{m}$ ) were de-paraffinized, rehydrated in a descending ethanol series and treated with $0.3 \% \mathrm{H}_{2} \mathrm{O}_{2}$. The sections were heated in $1 \%$ citrate buffer for $5 \mathrm{~min}$ at $90-100^{\circ} \mathrm{C}$ for antigen retrieval, and blocked with $10 \%$ normal goat serum (Gemini Bio-Products, Sacramento, CA, USA) for $1 \mathrm{~h}$ at room temperature. Subsequent to being washed, the sections were incubated with rabbit anti-ECT2 antibodies (cat. no. bs-4102R; 1:50 dilution; BIOSS, Beijing, China) $(19,20)$ overnight at $4^{\circ} \mathrm{C}$. The negative control sections were incubated with PBS. After being washed three times with PBST, the bound antibodies were detected with horseradish peroxidase-conjugated anti-IgG (cat. no. 111-035-144; 1:5,000 dilution; Jackson ImmunoResearch Laboratories, Inc., West Grove, PA, USA) and immunoreactive signals were developed using diaminobenzidine tetrachloride, followed by counterstaining with haematoxylin for $2 \mathrm{~min}$ at room temperature. The staining was observed in a light microscope (x200, magnification). The levels of ECT2 expression in 81 LSCC samples were semi-quantitative analyzed by two pathologists (Department of Pathology, The Affiliated Hospital of Southwest Medical University, Luzhou, China) in a blinded manner. Briefly, the staining intensities were scored as follows: 0, no staining; 1, weak staining; 2, moderate staining; and 3, strong staining (21). The percentage of positive cells was recorded as $0, \leq 5 \% ; 1,6-25 \% ; 2,25-50 \%$; and $3,>50 \%$. The final scores were calculated by combining the staining intensity score and the positive percentage score. A score of $>3$ was regarded as high expression, while anything $\leq 3$ was considered as low expression.

Statistical analysis. Data are presented as the mean \pm standard deviation. The experiments were repeated three times. SPSS statistical software package 23.0 (IBM Corp., Armonk, NY, USA) was applied in statistical analysis. The difference between groups was analyzed by paired Student's t-test, $\chi^{2}$ test or Fisher's exact test when applicable. Survival time was estimated by Kaplan-Meier method and analyzed by the log-rank test. The potential correlation of ECT2 mRNA transcripts and anti-ECT2 immunochemistry staining was analyzed by Pearson's correlation. $\mathrm{P}<0.05$ was considered to indicated a statistically significant difference.

\section{Results}

ECT2 expression was upregulated in LSCC tissues and correlated with poor differentiation of LSCC. To determine the potential role of ECT2 in the development and progression of LSCC, 81 patients with LSCC were recruited and their demographic and clinical characteristics are presented in Table I. The expression levels of ECT2 expression were examined by immunohistochemistry. As indicated in Fig. 1, ECT2 protein was primarily expressed in the cytoplasm of tumor cells. A limited expression of ECT2 was detected in the matched adjacent non-tumor tissues, while an upregulated ECT2 protein expression was detected in LSCC, particularly in the low differentiated tumor tissues $(\mathrm{P}=0.039)$. Stratification analysis indicated that the increased levels of ECT2 expression were significantly associated with the medium and low differentiation of LSCC $(\mathrm{P}=0.039$; Table I), increased TNM stages $(\mathrm{P}=0.016)$, increased overall stages $(\mathrm{P}=0.007)$ and lymph node metastasis $(\mathrm{P}=0.04)$; however, it was not 
Table I. Association of ECT2 expression with clinicopathological characteristics of patients with LSCC.

\begin{tabular}{|c|c|c|c|c|}
\hline \multirow[b]{2}{*}{ Clinicopathological characteristics } & \multirow[b]{2}{*}{$\mathrm{n}$} & \multicolumn{2}{|c|}{ ECT2 expression } & \multirow[b]{2}{*}{ P-value } \\
\hline & & High (\%) & Low $(\%)$ & \\
\hline Type of organization & & & & $<0.001$ \\
\hline LSCC tissue & 81 & $56(69.37)$ & $25(31.63)$ & \\
\hline Adjacent non-tumor tissue & 81 & $21(27.16)$ & $60(72.84)$ & \\
\hline Sex & & & & 0.553 \\
\hline Male & 79 & $55(69.62)$ & $24(30.38)$ & \\
\hline Female & 2 & $1(50.00)$ & $1(50.00)$ & \\
\hline Age (years) & & & & 0.109 \\
\hline$<60$ & 38 & $25(65.78)$ & $13(33.22)$ & \\
\hline $60-70$ & 35 & $25(71.43)$ & $7(28.57)$ & \\
\hline$>70$ & 8 & $6(75.00)$ & $2(25.00)$ & \\
\hline Degree of differentiation & & & & 0.039 \\
\hline High & 42 & $23(54.76)$ & $19(45.24)$ & \\
\hline Medium & 35 & $29(82.86)$ & $6(17.14)$ & \\
\hline Low & 4 & $4(100.00)$ & $0(0.00)$ & \\
\hline \multicolumn{5}{|l|}{ TNM staging (17) } \\
\hline Tis+T1 & 17 & $8(47.71)$ & $9(52.29)$ & 0.002 \\
\hline $\mathrm{T} 2$ & 32 & $21(65.63)$ & $11(34.47)$ & \\
\hline $\mathrm{T} 3$ & 21 & $18(86.87)$ & $3(13.33)$ & \\
\hline $\mathrm{T} 4$ & 11 & $9(81.82)$ & $2(18.18)$ & \\
\hline $\mathrm{Tis}+\mathrm{T} 1+\mathrm{T} 2$ & 49 & $29(59.18)$ & $20(40.82)$ & 0.016 \\
\hline $\mathrm{T} 3+\mathrm{T} 4$ & 32 & $27(84.38)$ & $5(15.62)$ & \\
\hline Lymph node metastasis & & & & 0.040 \\
\hline Yes & 22 & $19(86.36)$ & $3(13.64)$ & \\
\hline No & 59 & $37(62.17)$ & $22(37.29)$ & \\
\hline Smoking & & & & 0.492 \\
\hline Yes & 53 & $38(71.71)$ & $3(13.64)$ & \\
\hline No & 28 & $18(64.28)$ & $22(37.29)$ & \\
\hline Overall staging (17) & & & & 0.003 \\
\hline I & 17 & $8(44.71)$ & $9(55.29)$ & \\
\hline II & 30 & $19(63.33)$ & $20(37.67)$ & \\
\hline III & 23 & $20(86.95)$ & $2(23.05)$ & \\
\hline IV & 11 & $9(81.82)$ & $2(18.18)$ & \\
\hline $\mathrm{I}+\mathrm{II}$ & 47 & 27 (59.79) & $20(40.21)$ & 0.007 \\
\hline $\mathrm{III}+\mathrm{IV}$ & 34 & $29(85.29)$ & $5(14.71)$ & \\
\hline Glottic & 58 & $40(68.97)$ & $18(31.03)$ & 0.958 \\
\hline Supraglottic & 23 & $16(69.09)$ & $7(30.91)$ & \\
\hline
\end{tabular}

ECT2, epithelial cell transforming sequence 2; LSCC, laryngeal squamous cell carcinoma; TNM, Tumor-Node-Metastasis.

significantly associated with sex, age, primary tumor site and smoking status in this population (Table I).

To further validate the data, the expression levels of ECT2 mRNA transcripts were determined in 25 pairs of LSCC and matched non-tumor tissue samples by RT-qPCR. The relative expression levels of ECT2 mRNA transcripts in the LSCC tissues were significantly increased, compared with the matched adjacent non-tumor tissues ( $\mathrm{P}<0.01$; Fig. $2 \mathrm{~A})$. The relative expression levels of ECT 2 mRNA transcripts in 25 LSCC were positively correlated with the intensity of anti-ECT2 staining $(\mathrm{R}=0.938 ; \mathrm{P}<0.05$; Fig. $2 \mathrm{~B})$. Therefore, increased levels of ECT2 expression are associated with lower differentiation of LSCC and may contribute to the development and progression of poorly differentiated LSCC.

ECT2 expression was negatively correlated with overall survival time of LSCC patients. A total of 81 patients with LSCC were followed-up. Among them, seven cases $(8.4 \%)$ 


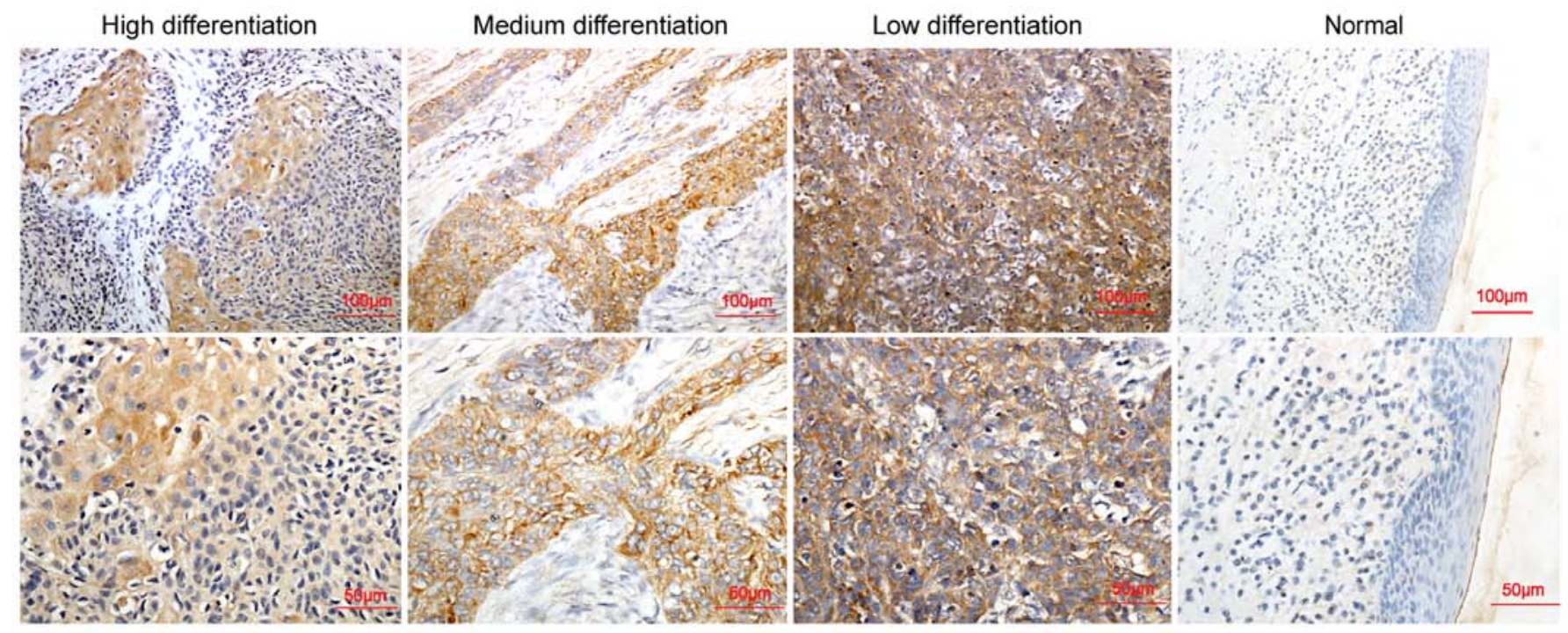

Figure 1. Immunohistochemistry analysis of epithelial cell transforming sequence 2 expression in laryngeal squamous cell carcinoma and matched adjacent nontumor tissues. Magnification, x200 for upper panels and x400 for bottom panels of each group of samples.
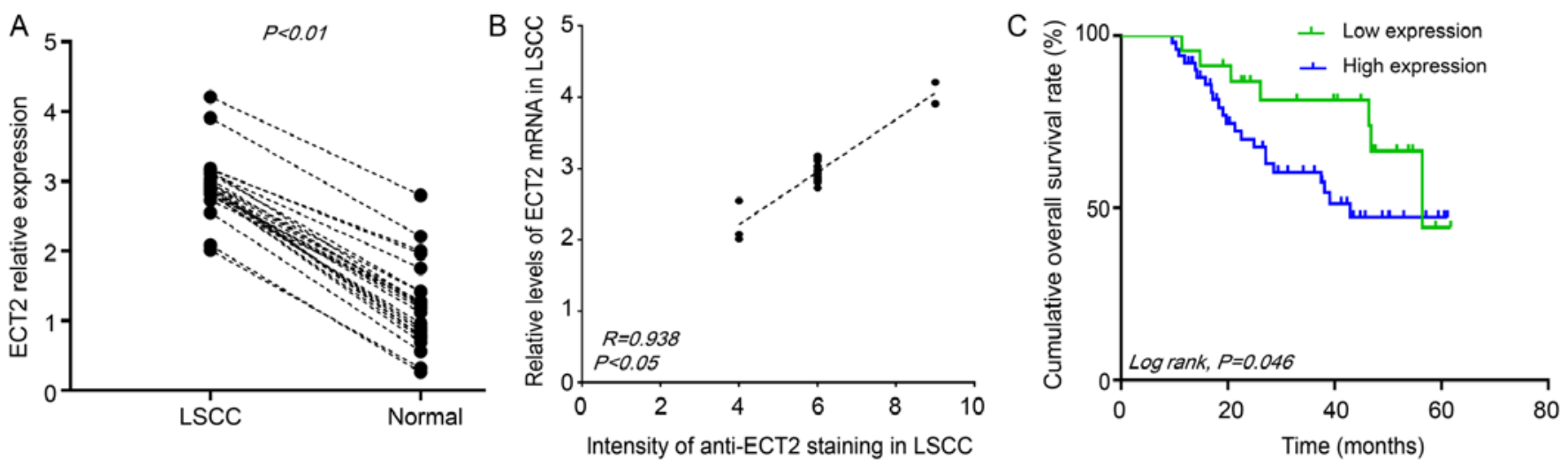

Figure 2. Upregulated ECT2 is associated with a reduced overall survival rate of patients with LSCC. (A) Analysis of ECT2 mRNA transcripts. The relative expression levels of ECT2 to GAPDH mRNA transcripts in 25 pairs of LSCC and adjacent nontumor tissues. Each experiment was performed in triplicate with $\mathrm{P}<0.01$ determined by paired Student's t-test. (B) The correlation between the relative expression levels of ECT2 mRNA transcripts and the intensities of anti-ECT2 staining in 25 ECT2+LSCC specimens determined by Pearson's correlation. (C) Survival analysis, based on the levels of ECT2 expression and patient overall survival rate. Patients with increased ECT2 expression, $n=56$; and patients with reduced ECT2 expression, n=25. ECT2, epithelial cell transforming sequence 2; LSCC, laryngeal squamous cell carcinoma.

were lost for follow-up, due to personal reasons. Stratification analysis indicated that the overall survival time period of patients with increased ECT2 expressing LSCC was significantly reduced, compared with those with reduced ECT2 expressing LSCC ( $\mathrm{P}=0.046$; Fig. 2C). Therefore, increased levels of ECT2 are associated with poor prognosis of LSCC.

\section{Discussion}

LSCC invasion and metastasis are leading causes of mortality (22). However, to the best of our knowledge, no reliable prognostic biomarkers for LSCC have been identified. ECT2 is physiologically expressed primarily in the nucleus and spreads into the cytoplasm of cells during mitosis (13). ECT2 can be misplaced in the cytoplasm, where ECT2 binds to the protein kinase $\mathrm{Cl} /$ polarity protein $6 \alpha(\mathrm{PKCl} / \mathrm{Par} 6 \alpha)$ complex and is phosphorylated by $\mathrm{PKC} \mathrm{C}$ to activate Rac family small GTPase 1 (Rac1), and promote the proliferation and invasion of non-small cell lung carcinoma (NSCLC) cells (23). Furthermore, $\mathrm{PKCl}$ or Par6 $\alpha$ silencing also promotes the redistribution of ECT2 from the cytoplasm to nucleus (24). Therefore, $\mathrm{PKCl} / \mathrm{Par} 6 \alpha$ complex in NSCLC regulates ECT2 cytoplasmic retention to induce phosphorylation and activation of downstream Rac1, conferring its oncogenic activity (24). In the present study, it was indicated that the upregulated ECT2 expression was primarily located in the cytoplasm of LSCC. This indicated that upregulated ECT2 expression in the cytoplasm may contribute to the development and progression of LSCC through similar mechanisms.

In the present study, it was indicated that upregulated ECT2 expression was associated with reduced differentiation, increased TNM stage, and lymph node metastasis of LSCC in the sample population. It has been reported that lowly differentiated tumors are associated with aggressive progression and poor prognosis in numerous malignant tumor types, including epithelial ovarian carcinoma, bladder 
squamous cell carcinoma and thyroid carcinoma (25-28). Because up-regulation of ECT2 and its misplacement are demonstrated in lowly differentiated LSCC, ECT2 may serve as a potential biomarker for the prognosis of LSCC.

Increased levels of ECT2 expression were associated significantly with poor overall survival time period of patients with LSCC. The data of the present study is in accordance to previous reports on other malignant tumors including lung cancer, colorectal cancer, and ovarian cancer $(15,16,29,30)$ and indicate that ECT2 may serve as an oncogenic factor to promote the progression of LSCC. Therefore, an increased ECT2 expression is a valuable biomarker for differentially diagnosis and prognosis of LSCC.

There are a number of limitations in the present study, including small sample size. Therefore, larger sample sizes are required in order to examine the potential molecular mechanisms underlying the action of ECT2 in promoting the progression of LSCC.

\section{Acknowledgements}

The authors thank their colleagues in Chongqing Medical University (Chongqing, China) and Southwest Medical University (Luzhou, China) for their suggestions and critical reading of the manuscript.

\section{Funding}

No funding was received.

\section{Availability of data and materials}

All data from this study are included in the article.

\section{Authors' contributions}

HYC, LY and LZJ were responsible for the conception and design of the present study. LZ, BL, TYW, YLL and TJZ collected and analyzed the data. LZ, GQ, BL and TJZ analyzed and interpreted the data. LZ wrote the manuscript. HYC, LY, GQ, LZJ, TJZ, BL, TYW and YLL critically revised the manuscript for important intellectual content. All authors gave intellectual input to the study and approved the final version of the manuscript.

\section{Ethics approval and consent to participate}

This study was approved by the Ethics Committee of the Affiliated Hospital of Southwest Medical University. All procedures performed in studies involving human participants were in accordance with the ethical standards of the institutional and national research committee and with the 1964 Helsinki declaration and its later amendments or comparable ethical standards. Written informed consent was obtained from all individual participants in the study.

\section{Patient consent for publication}

Patients provided written informed consent for the publication of the data.

\section{Competing interests}

The authors declare that they have no competing interests.

\section{References}

1. Ferlay J, Bray F, Pisani P and Parkin DM: GLOBOCAN 2000: Cancer incidence, mortality and prevalence worldwide, version 1.0. IARC CancerBase No. 5. Lyon, IARCPress, 2001.

2. Ramroth H, Dietz A and Becher H: Interaction effects and population-attributable risks for smoking and alcohol on laryngeal cancer and its subsites. A case-control study from Germany. Methods Inf Med 43: 499-504, 2004.

3. Shangina O, Brennan P, Szeszenia-Dabrowska N, Mates D, Fabiánová E, Fletcher T, t'Mannetje A, Boffetta P and Zaridze D: Occupational exposure and laryngeal and hypopharyngeal cancer risk in central and eastern Europe. Am J Epidemiol 164: 367-375, 2006.

4. Talamini R, Bosetti C, La Vecchia C, Dal Maso L, Levi F, Bidoli E, Negri E, Pasche C, Vaccarella S, Barzan L, et al: Combined effect of tobacco and alcohol on laryngeal cancer risk: a case-control study. Cancer Causes Control 13: 957-964, 2002.

5. Becher H, Ramroth H, Ahrens W, Risch A, Schmezer P and Dietz A: Occupation, exposure to polycyclic aromatic hydrocarbons and laryngeal cancer risk. Int J Cancer 116: 451-457, 2005.

6. Ramroth H, Dietz A, Ahrens W and Becher H: Occupational wood dust exposure and the risk of laryngeal cancer: a population based case-control study in Germany. Am J Ind Med 51: 648-655, 2008.

7. Nachalon Y, Cohen O, Alkan U, Shvero J and Popovtzer A: Characteristics and outcome of laryngeal squamous cell carcinoma in young adults. Oncol Lett 13: 1393-1397, 2017.

8. Scoumanne A and Chen X: The epithelial cell transforming sequence 2, a guanine nucleotide exchange factor for Rho GTPases, is repressed by $\mathrm{p} 53$ via protein methyltransferases and is required for G1-S transition. Cancer Res 66: 6271-6279, 2006.

9. Miki T, Smith CL, Long JE, Eva A and Fleming TP: Oncogene ect2 is related to regulators of small GTP-binding proteins. Nature 362: 462-465, 1993.

10. Yüce O, Piekny A and Glotzer M: An ECT2-centralspindlin complex regulates the localization and function of RhoA. J Cell Biol 170: 571-582, 2005.

11. Tatsumoto T, Xie X, Blumenthal R, Okamoto I and Miki T: Human ECT2 is an exchange factor for Rho GTPases, phosphorylated in G2/M phases, and involved in cytokinesis. J Cell Biol 147: 921-928, 1999.

12. Sano M, Genkai N, Yajima N, Tsuchiya N, Homma J, Tanaka R, Miki T and Yamanaka R: Expression level of ECT2 proto-oncogene correlates with prognosis in glioma patients. Oncol Rep 16: 1093-1098, 2006.

13. Saito S, Liu XF, Kamijo K, Raziuddin R, Tatsumoto T, Okamoto I, Chen X, Lee CC, Lorenzi MV, Ohara N, et al: Deregulation and mislocalization of the cytokinesis regulator ECT2 activate the Rho signaling pathways leading to malignant transformation. J Biol Chem 279: 7169-7179, 2004.

14. Matthews HK, Delabre U, Rohn JL, Guck J, Kunda P and Baum B: Changes in Ect2 localization couple actomyosin-dependent cell shape changes to mitotic progression. Dev Cell 23: 371-383, 2012.

15. Luo Y, Qin SL, Mu YF, Wang ZS, Zhong M and Bian ZQ: Elevated expression of ECT2 predicts unfavorable prognosis in patients with colorectal cancer. Biomed Pharmacother 73: 135-139, 2015.

16. Murata Y, Minami Y, Iwakawa R, Yokota J, Usui S, Tsuta K, Shiraishi K, Sakashita S, Satomi K, Iijima T, et al: ECT2 amplification and overexpression as a new prognostic biomarker for early-stage lung adenocarcinoma. Cancer Sci 105: 490-497, 2014.

17. Patel SG and Shah JP: TNM staging of cancers of the head and neck: Striving for uniformity among diversity. CA Cancer J Clin 55: 242-258, quiz 261-262, 264, 2005.

18. Livak KJ and Schmittgen TD: Analysis of relative gene expression data using real-time quantitative PCR and the 2(-Delta Delta C(T)) Method. Methods 25: 402-408, 2001.

19. Wang HB, Yan HC and Liu Y: Clinical significance of ECT2 expression in tissue and serum of gastric cancer patients. Clin Transl Oncol 18: 735-742, 2016.

20. Guo Z, Chen X, Du T, Zhu D, Lai Y, Dong W, Wu W, Lin C, Liu L and Huang H: Elevated levels of epithelial cell transforming sequence 2 predicts poor prognosis for prostate cancer. Med Oncol 34: 13, 2017. 
21. Rizzardi AE, Johnson AT, Vogel RI, Pambuccian SE, Henriksen J, Skubitz AP, Metzger GJ and Schmechel SC: Quantitative comparison of immunohistochemical staining measured by digital image analysis versus pathologist visual scoring. Diagn Pathol 7: 42, 2012.

22. Ferlay J, Soerjomataram I, Ervik M, Dikshit R, Eser S, Mathers C Rebelo M, Parkin DM, Forman D, Bray F (Eds.): GLOBOCAN 2012 v1.0, Cancer incidence and mortality worldwide: IARC CancerBase No. 11. International Agency for Research on Cancer, Lyon, France, 2013.

23. Justilien V, Jameison L, Der CJ, Rossman KL and Fields AP: Oncogenic activity of Ect 2 is regulated through protein kinase $C$ iota-mediated phosphorylation. J Biol Chem 286: 8149-8157, 2011.

24. Justilien V and Fields AP: Ect2 links the PKCiota-Par6alpha complex to Rac1 activation and cellular transformation. Oncogene 28: 3597-3607, 2009.

25. Vergote I, De Brabanter J, Fyles A, Bertelsen K, Einhorn N, Sevelda P, Gore ME, Kaern J, Verrelst H, Sjövall K, et al: Prognostic importance of degree of differentiation and cyst rupture in stage I invasive epithelial ovarian carcinoma. Lancet 357: 176-182, 2001.
26. Ostergaard M, Rasmussen HH, Nielsen HV, Vorum H, Orntoft TF, Wolf $\mathrm{H}$ and Celis JE: Proteome profiling of bladder squamous cell carcinomas: Identification of markers that define their degree of differentiation. Cancer Res 57: 4111-4117, 1997.

27. Pollina L, Pacini F, Fontanini G, Vignati S, Bevilacqua $G$ and Basolo F: bcl-2, p53 and proliferating cell nuclear antigen expression is related to the degree of differentiation in thyroid carcinomas. Br J Cancer 73: 139-143, 1996.

28. Elston CW and Ellis IO: Pathological prognostic factors in breast cancer. I. The value of histological grade in breast cancer: Experience from a large study with long-term follow-up. Histopathology 19: 403-410, 1991.

29. Cook DR, Rossman KL and Der CJ: Rho guanine nucleotide exchange factors: Regulators of Rho GTPase activity in development and disease. Oncogene 33: 4021-4035, 2014.

30. Mack NA and Georgiou M: The interdependence of the Rho GTPases and apicobasal cell polarity. Small GTPases 5: 10, 2014. 